\title{
AUTOESTIMA Y COMPROMISO ORGANIZACIONAL EN TRABAJADORES DE UNA UNIVERSIDAD PÚBLICA DE PROVINCIAS
}

\author{
Alejandro Loli P. ${ }^{1}$, Edgardo Cuba B. ${ }^{2}$ \\ (Recibido el 04/02/2007, aceptado el 28/03/2007)
}

\begin{abstract}
RESUMEN
El estudio intenta conocer la relación que existe entre la autoestima y el compromiso organizacional de los trabajadores administrativos de una universidad pública de provincia, en una muestra de cuarenta y ocho sujetos que representa el $18 \%$ de la población total. El estudio arroja que no existe relación entre autoestima y compromiso organizacional; sin embargo, encontramos que la autoestima está relacionada con los factores del compromiso organizacional, especialmente compromiso con el trabajo.
\end{abstract}

Palabras clave: Autoestima, Compromiso organizacional, Trabajadores.

\begin{abstract}
The study attempts to examine the relationship that exists between self-esteem and the organizational commitment of administrative workers within a provincial public university using a sample of 48 subjects, representing $18 \%$ of the total university population. The study shows that a relationship between self-esteem and organizational commitment does not exist; however, we found that a correlation exists between self-esteem and factors related to organizational commitment, specifically commitment to work.
\end{abstract}

Keywords: Self-esteem, Organizational commitment, Workers.

\section{INTRODUCCIÓN}

En los últimos 15 años, el Perú está afrontando cambios sustanciales tanto en lo económico como en lo político, social y cultural, esencialmente a partir de la apertura de las fronteras a la globalización del mundo. La importación de la tecnología y su implantación en el mercado nacional, la inversión privada internacional, entre otras, han llevado al crecimiento de la economía del país, permitiendo tener un concepto diferente del mundo. Sin embargo, las organizaciones encuentran que su crecimiento no es suficiente.

1 Docente Principal, Facultad de Psicología de la UNMSM. E-mail: alolip@unmsm.edu.pe

2 Docente Asociado, Facultad de Psicología de la UNMSM. E-mail: edlcubab@yahoo.es 
Las grandes organizaciones internacionales y algunas nacionales tienen conciencia clara de que el dinero y la tecnología efectivamente no podrán responder de manera efectiva a las exigencias de una sociedad en tanto no se revalorice la intervención del capital humano. Al respecto, se han desarrollado diversas investigaciones, todas ellas vinculadas a factores humanos (valores, compromiso, clima, satisfacción, autoestima, entre otros) que puedan estar influyendo en el rendimiento de los trabajadores para el desarrollo efectivo de las organizaciones. T. Peters y R.H. Waterman (1982) estudiaron sobre los valores, encontrando que la mayoría de las características de las organizaciones exitosas se basan en los valores humanos, como prioridad de sus acciones. J. Cruz (1996) y M. Rodríguez (1993) consideran que la autoestima y los valores organizacionales son importantes en el éxito personal y organizacional.

En 1998, Alejandro E. Loli Pineda y colaboradores realizaron una investigación relativa a la autoestima y los valores organizacionales en 105 gerentes de Microempresas, encontrando correlación significativa entre autoestima y algunos valores organizacionales (lealtad, amor al trabajo, trabajo en equipo), y también autoestima con algunas variables demográficas. Por otro lado, estos autores (2000) estudian estas mismas variables en una muestra de 1,464 estudiantes universitarios de Lima, encontrando que existe asociación significativa entre la autoestima y los valores organizacionales (limpieza, responsabilidad, respeto a la persona).

Respecto al compromiso organizacional, F. Arias Galicia, D. Valera, A. Loli Pineda (2003), realizaron un estudio en una muestra de 167 personas, la mayoría de educación superior, provenientes de diversas organizaciones y tamaños de ambos sexos, llegando a la conclusión que existe correlación significativa entre el compromiso afectivo y diversas variables organizacionales (antigüedad en el puesto, claridad del rol, compromiso con el trabajo, satisfacción general con el trabajo), así como el compromiso de conveniencia que se correlaciona con claridad del rol, compromiso con el trabajo, satisfacción general con el trabajo. Estos resultados expresan que el compromiso organizacional es tan importante como la autoestima para el logro de éxitos organizacionales.

Una investigación reciente de Alejandro E. Loli Pineda (2005) en una muestra de 205 trabajadores de una universidad pública de Lima, arroja una tendencia favorable de los trabajadores hacia el compromiso organizacional, y el análisis de los factores nos indica correlación muy significativa y positiva entre satisfacción general con el trabajo y compromiso con el trabajo, compromiso afectivo, jerarquía del puesto y sentimiento de permanencia; mientras que, la relación entre el primero y el compromiso de conveniencia es muy significativa pero negativa. Igual ocurre entre el compromiso con el trabajo y compromiso de conveniencia. También encontramos asociación muy significativa y positiva entre el compromiso con el trabajo y el compromiso afectivo, sentimiento de permanencia y jerarquía del puesto.

Por consiguiente, la probabilidad que exista correlación entre el compromiso organizacional y la autoestima es preocupación del presente estudio, así como el compromiso organizacional y algunas variables demográficas. 


\section{METODO}

\section{Muestra}

La muestra estuvo constituida por 48 trabajadores de la Universidad Nacional Agraria de la Selva, en el interior del país; aunque los instrumentos fueron aplicados en un número mayor, éstos no llenaron los datos completos, por lo que se tuvo que reducir al $18 \%$ de la población total. De los cuales $18,75 \%$ son mujeres y el $81,25 \%$ son varones. Una característica común de la población, aunque coyuntural, fue que salían de un conflicto laboral (huelga de 2 meses), donde los maltratos y entredichos entre ellos y entre las autoridades fue muy dura, provocando polarizaciones entre los grupos, resentimientos y malos entendidos, situación que puede tener relevancia en la asociación de la autoestima con el compromiso organizacional de los trabajadores.

\section{Técnica de recolección de datos}

La técnica empleada para la captura de la información fue mediante la administración colectiva en un auditorio de la aniversidad a la culminación de un ciclo de conferencias. Dicha información se logró con el apoyo de un funcionario de la Oficina de Recursos Humanos que fue instruido para la administración de los instrumentos. Cuyos formatos obviamente no fueron revisados al ser recogidos o se negaron a llenar en su totalidad.

\section{Instrumento}

Los instrumentos que sirvieron de filtro para la recolección de la información fueron: el Inventario de Autoestima-ALPEL- Forma AD y el cuestionario de Compromiso Organizacional. El primero, construido y validado por Alejandro E. Loli P. y Ernestina López V. (2005), en una muestra de 3215 sujetos (2785 estudiantes universitarios y 430 sujetos egresados de universidades y profesionales). El análisis de consistencia de los ítemes arroja un coeficiente de confiabilidad Kuder Richardson 20 de 0.84, altamente confiable. El segundo fue construido por el Dr. Fernando Arias Galicia (2003) sobre la base de investigaciones realizadas por otros autores (Meyer y Allen, 1991: Compromiso afectivo y de Conveniencia; Eisenberger, 1997: Satisfacción general con el trabajo; Brown y Leigh, 1996 : Claridad del Rol; Lodahl y Kejner, 1965: Compromiso con el trabajo).

\section{Procesamiento de datos}

Se empleó el SPSS, versión 11.5. Con el objeto de verificar la asociación entre la autoestima y el compromiso organizacional, se utilizó el coeficiente de correlación de Spearman porque los puntajes de autoestima y compromiso organizacional no siguieron un comportamiento normal. Luego, se procedió a comparar los grupos demográficos mediante el puntaje «t»y ANOVA, no obteniendo resultado alguno, podría ser debido a lo reducido de la muestra y la variedad de los grupos de análisis. 


\section{RESULTADOS}

\section{La autoestima y el compromiso organizacional general}

En la tabla 1 se observa la correlación entre la autoestima y el compromiso organizacional de los trabajadores de la entidad estudiada. En términos generales, los resultados indican que no existe asociación entre la autoestima y el compromiso organizacional p (sig.2-tailed $=0.544$ ), de acuerdo con el coeficiente de correlación de Spearman. Vale decir, que la autoestima de los trabajadores es independiente en relación con el compromiso que pueda existir con la organización. Esto nos indica que probablemente el respeto, la confianza, el conocimiento y la realización personal de los trabajadores están disminuidos o individualizados, que no están interesados en correr riesgos, o no son capaces de asumir compromisos que los afecten, sobre todo, con la organización donde trabajan.

Tabla 1. Correlación ente autoestima y compromiso organizacional en muestra total.

\begin{tabular}{llcc}
\hline & & $\begin{array}{c}\text { Puntaje } \\
\text { autoestima }\end{array}$ & $\begin{array}{c}\text { Compromiso } \\
\text { total }\end{array}$ \\
\hline Puntaje & Coefic. Correlación & 1.000 & .090 \\
autoestima & Sig. & 48 & .544 \\
& $\mathrm{~N}$ & .090 & 48 \\
Compromiso & Coefic. Correlación & .544 & 1.000 \\
total & Sig. & 48 & 48 \\
\hline
\end{tabular}

\section{La autoestima y los factores del compromiso organizacional}

En la tabla 2 observamos la correlación de Spearman entre el puntaje de la autoestima y los puntajes de los factores del compromiso organizacional. Los resultados nos muestran que no existe relación entre la autoestima y la satisfacción general con el trabajo $(\mathrm{p}<0.848)$, el compromiso de conveniencia $(\mathrm{p}<0.357)$, el compromiso afectivo $(\mathrm{p}<0.372)$, el sentimiento de permanencia $(\mathrm{p}<0.223)$ y la jerarquía del puesto $(\mathrm{p}<0.571)$.

En cambio, encontramos que la autoestima de los trabajadores está asociada al compromiso con el trabajo $(\mathrm{p}<0.013)$, y esta asociación es positiva. Lo que significa que a mayor autoestima mayor compromiso con el trabajo; es decir, parece no existir ningún otro elemento que los vincule más que con su trabajo en concreto.

Es posible que, debido a la escasez de fuentes de trabajo, a la inseguridad experimentada en el centro de trabajo durante el conflicto, al deterioro de la imagen de la institución, entre otras razones, los trabajadores no tienen interés por un vínculo diferente y mayor.

Tabla 2. Correlación entre autoestima y factores de compromiso organizacional.

\begin{tabular}{cccc}
\hline FACTORES & r Spearman & p-value & significancia \\
\hline PUA vs ST & 0.028 & 0.848 & n.s \\
PUA vs CT & 0.357 & 0.013 & * \\
PUA vs CC & -0.136 & 0.357 & n.s \\
PUA vs CA & 0.132 & 0.372 & n.s \\
PUA vs SP & 0.179 & 0.223 & n.s \\
PUA vs JP & 0.084 & 0.571 & n.s \\
\hline
\end{tabular}




\section{DISCUSIÓN}

A la luz de los resultados, se puede decir que en la muestra estudiada no existe asociación entre la autoestima y el compromiso organizacional de los trabajadores, contraria a la hipótesis de que esa asociación es posible en tanto la autoestima tiene un contenido afectivo, «... Si una persona se conoce y está conciente de sus cambios, crea su propia escala de valores y desarrolla sus capacidades; y si se acepta y respeta, tendrá autoestima...» (M. Rodríguez, 1993), si ocurre lo contrario la autoestima estará disminuida. J. A. Alcántara (1993) señala que la autoestima «... es una actitud hacia uno mismo... es la disposición permanente según la cual nos enfrentamos con nosotros mismos...». Entonces, una alteración de nuestras actitudes hacia uno mismo, por razones internas o externas, puede alterar nuestra percepción respecto a los demás, por tanto, nuestro comportamiento. La autoestima es energía, es pasión y es visión, luego, vulnerable a cambios de acuerdo a las circunstancias.

Así visto, y teniendo como antecedentes el conflicto laboral y organizacional en el que estaban envueltos los sujetos estudiados, se explica el porqué no es posible la asociación entre la autoestima y el compromiso organizacional; pero también puede estar demostrando que la autoestima es más individual que organizacional, pero que sí afecta a la organización como conjunto.

Sin embargo, revisando la asociación con cada uno de los componentes del compromiso organizacional, vemos que existe asociación de la autoestima con compromiso con el trabajo y no así con los demás componentes. Este resultado es coherente con lo encontrado por Alejandro E. Loli Pineda (1998) donde afirman que existe asociación entre la autoestima y los valores organizacionales tales como amor al trabajo, lealtad y trabajo en equipo, en una muestra de gerentes. Pero también, en una muestra más amplia de estudiantes universitarios, el mismo autor (2000) encuentra asociación de la autoestima con valores organizacionales como limpieza, responsabilidad y respeto a la persona. Situación que hace pensar, que la autoestima, sin dejar de ser más individual que organizacional, compromete a la organización como conjunto porque afecta los valores organizacionales.

Igualmente, estudios realizados por F. Arias Galicia, D. Valera, A. Loli Pineda (2003) en 167 sujetos, la mayoría compuesto por estudiantes de educación superior, sobre compromiso organizacional, demuestran que hay asociación del compromiso afectivo y compromiso de conveniencia (componentes del compromiso organizacional) con compromiso con el trabajo y otras variables. Resultado éste que es confirmado por A. Loli Pineda (2005) en un estudio similar, esta vez en una muestra de 205 trabajadores de una universidad pública de Lima.

Por consiguiente, es posible que una muestra más amplia sobre el tema pueda arrojar resultados diferentes. Naturalmente, habría que cuidar que los instrumentos sean administrados en organizaciones donde la autoestima de los trabajadores no esté afectada por conflictos internos de la organización. Hecho que podría demostrar que efectivamente la autoestima es sensible a los acontecimientos externos del ambiente y, sobre todo, confirmar o rechazar los resultados de la presente investigación.

\section{CONCLUSIONES}

1. En términos generales, no existe asociación entre la autoestima y el compromiso organizacional de los trabajadores. Vale decir, que la autoestima de los trabajadores es independiente en relación con el compromiso que pueda existir con la organización.

2. Una exploración con cada uno de los componentes del compromiso organizacional, indica que la autoestima de los trabajadores está asociada sólo con el compromiso con 
el trabajo, y esta asociación es positiva. Lo que significa que a mayor autoestima mayor compromiso con el trabajo; es decir, parece no existir ningún otro elemento que los vincule más que con su trabajo en concreto.

3. Existe la posibilidad, que un estudio en una muestra más amplia y en organizaciones donde se supone no existen conflictos y que la autoestima de los sujetos investigados se mantiene estable, arroje resultados diferentes.

\section{REFERENCIAS BIBLIOGRÁFICAS}

1 Antón, C. y Gonzáles, L. (2005). Examen de las relaciones entre el compromiso organizacional afectivo y continuo. España: Universidad de Salamanca.

2 Arias Galicia, Fernando (2001). El compromiso personal hacia la organización y la intención de permanencia: algunos factores para su incremento. Rev. De contaduría y administración $\mathrm{N}^{\circ}$ 200, enero-marzo 2001. México: Edit. UNAM.

3 Arias Galicia, F.; Loli Pineda., A.; y col. (2003). El compromiso organizacional y su relación con algunos factores demográficos y psicológicos. Revista Investigación Psicológica. Lima: Instituto de Investigaciones Psicológicas, UNMSM.

4 Bayona, C., Goñi, S. y Madorrán, C. (2000). Compromiso organizacional: implicaciones para la gestión estratégica de los recursos humanos. www.upna.es

5 Bonet, José (1994). Sé amigo de ti mismo, Manual de autoestima. Bilbao: Edit. Sal Térrea.

6 Claure Rocha, M. y Böhrt Pelaez, M. (2003). Tres dimensiones del compromiso organizacional: identificación, membresía y lealtad. Rev. $A J A Y U$, año 2003, Vol. 2, $\mathrm{N}^{\mathrm{o}}$ 1. Bolivia. Estas dimensiones son equivalentes a compromiso afectivo, continuidad y normativo de Meyer y Allen (1991), respectivamente.

7 Cruz Ramírez, José (1997). Autoestima y gestión de la calidad. México: Edit. Iberoamericana.

8 Lindenfield, Gael (1998). Cómo desarrollar la autoestima en niños y adolescentes. Madrid: Editorial Neo Person.

9 Loli Pineda, Alejandro (2005). Inventario de autoestima para adultos (ALPEL) Forma $A D$. Lima: Edit. Celtronic del Perú SAC.

10 Loli Pineda, Alejandro (2005). «Compromiso organizacional de los trabajadores y su relación con algunas variables demográficas». Instituto de Investigaciones Psicológicas, UNMSM, Lima, Perú.

11 Loli Pineda, Alejandro (2000). La autoestima y los valores organizacionales en estudiantes universitarios de Lima. Lima: Instituto de Investigaciones Psicológicas, UNMSM.

12 Loli Pineda, Alejandro y López Vega, Ernestina (1998). «Autoestima y valores en la calidad y la excelencia». Lima: Instituto de Investigaciones Psicológicas, UNMSM.

13. Loli Pineda, A. y López Vega, E. (1998). La autoestima y los valores en las empresas de Lima. Lima: Instituto de Investigaciones Psicológicas, UNMSM.

14. Meyer, J. P. y Allen, N. J. (1991). Tres componentes, Conceptualización del compromiso organizacional. Human Resource Management Review 1, 61-98.

15. Peters T. y Waterman R. (1996). En busca de la excelencia. Colombia: Editorial Norma.

16. Rodríguez Estrada, Mauro (1993). Los valores, clave de la excelencia. México: Editorial McGraw Hill. 